\title{
Steady-State VEP-Based Brain-Computer Interface Control in an Immersive 3D Gaming Environment
}

\author{
E. C. Lalor, ${ }^{1}$ S. P. Kelly, ${ }^{1,2}$ C. Finucane, ${ }^{3}$ R. Burke, ${ }^{4}$ R. Smith, ${ }^{1}$ R. B. Reilly, ${ }^{1}$ and G. McDarby ${ }^{1}$ \\ ${ }^{1}$ School of Electrical, Electronic and Mechanical Engineering, University College Dublin, Belfield, Dublin 4, Ireland \\ Emails:ed.lalor@ee.ucd.ie,ray.smith@ee.ucd.ie,richard.reilly@ucd.ie,gary.mcdarby@ee.ucd.ie \\ ${ }^{2}$ The Cognitive Neurophysiology Laboratory, Nathan S. Kline Institute for Psychiatric Research, Orangeburg NY 10962, USA \\ Email: skelly@nki.rfmh.org, \\ ${ }^{3}$ Medical Physics and Bioengineering, St. James's Hospital, P.O. Box 580, Dublin 8, Ireland \\ Email: cfinucane@stjames.ie \\ ${ }^{4}$ EOC Operations Center, Microsoft Corporation, Sandyford Industrial Estate, Dublin 18, Ireland \\ Email: robert.burke@gmail.com
}

Received 2 February 2004; Revised 19 October 2004

\begin{abstract}
This paper presents the application of an effective EEG-based brain-computer interface design for binary control in a visually elaborate immersive 3D game. The BCI uses the steady-state visual evoked potential (SSVEP) generated in response to phasereversing checkerboard patterns. Two power-spectrum estimation methods were employed for feature extraction in a series of offline classification tests. Both methods were also implemented during real-time game play. The performance of the BCI was found to be robust to distracting visual stimulation in the game and relatively consistent across six subjects, with 41 of 48 games successfully completed. For the best performing feature extraction method, the average real-time control accuracy across subjects was $89 \%$. The feasibility of obtaining reliable control in such a visually rich environment using SSVEPs is thus demonstrated and the impact of this result is discussed.
\end{abstract}

Keywords and phrases: EEG, BCI, SSVEP, online classification, overt attention.

\section{INTRODUCTION}

The concept of a brain-computer interface (BCI) stems from a need for alternative, augmentative communication, and control options for individuals with severe disabilities (e.g., amyotropic lateral sclerosis), though its potential uses extend to rehabilitation of neurological disorders, brain-state monitoring, and gaming [1]. The most practical and widely applicable BCI solutions are those based on noninvasive electroencephalogram (EEG) measurements recorded from the scalp. These generally utilize either event-related potentials (ERPs) such as P300 [2] and visual evoked potential (VEP) measures [3], or self-regulatory activity such as slow cortical potentials [4] and changes in cortical rhythms $[5,6,7]$. The former design, being reliant on natural involuntary responses, has the advantage of requiring no training, whereas the latter design normally demonstrates effectiveness only after periods of biofeedback training, wherein the subject learns to regulate the relevant activity in a controlled way.

Performance of a BCI is normally assessed in terms of information transfer rate, which incorporates both speed and accuracy. One BCI solution that has seen considerable success in optimizing this performance measure relies on steady-state visual evoked potentials (SSVEPs), a periodic response elicited by the repetitive presentation of a visual stimulus at a rate of $6-8 \mathrm{~Hz}$ or more [8]. SSVEPs have been successfully utilized in both above-mentioned BCI designs-gaze direction within a matrix of flickering stimuli is uniquely manifest in the evoked SSVEP through its matched periodicity $[3,9]$, and also the self-regulation of SSVEP amplitude has been reported as feasible with appropriate feedback [10].

The effectiveness of SSVEP-based BCI designs is due to several factors. The signal itself is measurable in as large a population as the transient VEP-very few fail to exhibit this type of response $[8,11]$. The task of feature extraction is reduced to simple frequency component extraction, as there are only a certain number of separate target frequencies, usually one for each choice offered in the BCI. High signal-tonoise ratios are obtainable when analyzing the SSVEP at sufficiently high frequency resolution [8]. Finally, SSVEPs are resilient to artifacts, as blink, movement, and electrocardiographic artifacts are confined mostly to lower EEG frequencies [11]. Moreover, the source of ocular artifacts (blinks, eye 
movements) is located on the opposite side of the head to the visual cortex over which the SSVEP is measured. Though these characteristics are well affirmed by the success of current SSVEP-based BCIs [3, 9], it is not known to what degree performance may be compromised by concurrent unrelated visual stimulation, where an individual's visual resources are divided, as in a video gaming environment.

In this paper, the authors wish to address a novel application of the SSVEP-based BCI design within a real-time gaming framework. The video game involves the movement of an animated character within a virtual environment. Both the character and environment have been modelled as 3D volumes. The lighting and virtual camera position change in response to the character's movements within the environment. Overall, the result is a very visually engaging video game.

The SSVEP response constitutes only a portion of the overall set of visual processes manifest in the ongoing EEG during game play. In this study, we address the challenge of extracting and processing SSVEP measures from a signal of such indeterminate complexity in real time for BCI control.

The design of the SSVEP-based BCI was split into two parts. First, a preliminary offline analysis was conducted to determine the most favourable signal processing methodology and choose suitable frequencies. Once satisfactory offline analysis results were obtained, the full real-time game was implemented. Performance of the real-time BCI game when played by six normal subjects is presented.

\section{PRELIMINARY ANALYSIS}

\subsection{Methods}

\section{(A) Subjects}

Five male subjects, aged between 23 and 27, participated in the preliminary study. All subjects had normal or correctedto-normal vision.

\section{(B) Experimental setup}

Subjects were seated $70 \mathrm{~cm}$ from a $43 \mathrm{~cm}$ (“17”) computer monitor. EEG was acquired in a shielded room from two Ag$\mathrm{AgCl}$ scalp electrodes placed at sites $\mathrm{O} 1$ and $\mathrm{O}$, according to the 10-20 international electrode-positioning standard [12], situated over the left and right hemispheres of the primary visual cortex, respectively. Skin-electrode junction impedances were maintained below $5 \mathrm{k} \Omega$. Each channel, referenced to the right ear lobe on bipolar leads, was amplified $(20 \mathrm{~K})$, $50 \mathrm{~Hz}$ line filtered, and bandpass filtered over the range 0.01$100 \mathrm{~Hz}$ by Grass Telefactor P511 rack amplifiers. Assuming that eye movement and blink artifacts did not threaten signal integrity at frequencies of interest, neither horizontal nor vertical EOG signals were recorded. Subjects were monitored visually throughout for continued compliance. Signals were digitized at a sampling frequency of $256 \mathrm{~Hz}$.

Initial testing of the experimental setup involved acquiring data from two subjects while gazing at either a circular yellow flicker stimulus on black background or a similarly sized rectangular black and white checkerboard pattern, modulated at several test frequencies between $6 \mathrm{~Hz}$ and
$25 \mathrm{~Hz}$. On visual inspection of power spectra, it was found that the checkerboard pattern produced a more pronounced SSVEP than a flicker stimulus modulated at the same frequency. Furthermore, it has been found that to elicit an SSVEP signal at a certain frequency, a flicker stimulus must be modulated at that frequency, while a checkerboard pattern need only be modulated at half that frequency, as the SSVEP is produced at its rate of phase-reversal or alternation rate [13]. This is an important consideration when using a standard monitor with refresh rate of $100 \mathrm{~Hz}$. Hence, checkerboard patterns were chosen as stimuli in the following preliminary tests and BCI game. From this point, checkerboard frequencies will be given in terms of alternation rate, equivalent to the frequency of the SSVEP produced.

Twenty five seconds of eyes-closed data were first acquired for each subject to accurately locate alpha frequency. Testing then proceeded with several 25 -second trials during which the subject viewed a full-screen checkerboard pattern at frequencies between $6 \mathrm{~Hz}$ and $25 \mathrm{~Hz}$, excluding the individual's alpha band [9]. The power spectra for these data were examined and the two frequencies eliciting the largest SSVEPs were identified. The subject then underwent 25second trials in which he viewed each one of two bilateral checkerboard patterns phase-reversing at the two selected frequencies and this was repeated with positions reversed, giving a total of 4 trials. Each $4 \times 4$ checkerboard pattern's medial edge was situated $4.9^{\circ}$ bilateral to a central cross, centered on the horizontal meridian, and subtended a visual angle of $6.5^{\circ}$ vertically and $7.2^{\circ}$ horizontally. These dimensions were determined empirically.

\section{(C) Feature extraction}

Two feature extraction methods were employed for comparison in the preliminary data. Each was aimed at utilizing the separable aspects of the SSVEP signals. For both methods, each 25 -second trial was divided into approximately 50 overlapping segments, each of which counts as a single case for which the feature(s) is derived. Both 1-second and 2-second segments were used for comparison, with a view to assessing speed achievable by using each method in real time.

\section{Method 1: squared 4-second FFT}

In this method, each one- or two-second segment was extracted using a Hamming window, zero-padded to 1024 samples (4s), and the fast Fourier transform (FFT) was calculated and squared. A single feature was extracted for each segment:

$$
F 1(n)=\log \left(\frac{X_{n}(f 1)}{X_{n}(f 2)}\right)
$$

where

$$
X_{n}=\operatorname{mean}^{2}\left(\left.\operatorname{FFT}\left(x_{n}(t)\right)\right|_{O 1},\left.\operatorname{FFT}\left(x_{n}(t)\right)\right|_{O 2}\right),
$$

that is, the square of the FFT averaged over electrode sites $O 1$ and $O 2$, of the $n$th segment $x_{n}(t)$, and $f 1$ and $f 2$ are the chosen checkerboard frequencies. 


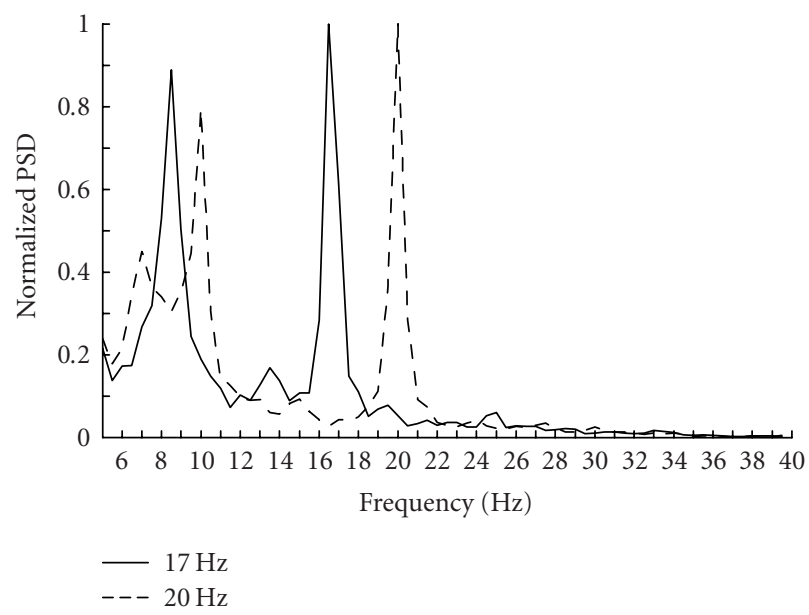

Figure 1: Power spectra for full-screen checkerboard trials at 17 and $20 \mathrm{~Hz}$ for subject 1 . Spectra were calculated using the squared FFT method averaged across the entire 25 -second trial.

\section{Method 2: FFT of autocorrelation}

This method is similar in that it also corresponds to calculating a PSD estimate. In this case, the autocorrelation function is calculated for each segment followed by the FFT:

$$
F 2(n)=\log \left(\frac{Y_{n}(f 1)}{Y_{n}(f 2)}\right)
$$

where

$$
\begin{gathered}
Y_{n}=\operatorname{mean}\left(\left.\operatorname{FFT}\left(R_{x x}{ }^{n}\right)\right|_{O 1},\left.\operatorname{FFT}\left(R_{x x}{ }^{n}\right)\right|_{O 2}\right), \\
R_{x x}{ }^{n}(t)=E\left\{x_{n}\left(t_{0}\right) x_{n}\left(t_{0}-t\right)\right\},
\end{gathered}
$$

where the second formula in (4) is the autocorrelation function of the $n$th segment $x_{n}(t)$.

This method of PSD estimation is more resilient to noise due to the fact that the autocorrelation of white noise is zero at all nonzero latencies.

\section{(D) Classification}

Linear discriminants were used as the classifier model for this study, providing a parametric approximation to Bayes' rule [14]. In the case of both feature extraction methods, this corresponds to calculating a threshold in one dimension. Optimization of the linear discriminant model is achieved through direct calculation and is very efficient, thus lending itself well to real-time applications.

Performance of the LDA classifier was assessed on the preliminary data using 10-fold cross-validation [14]. This scheme randomly divides the available data into 10 approximately equal-sized, mutually exclusive "folds." For a 10 -fold cross-validation run, 10 classifiers are trained with a different fold used each time as the testing set, while the other 9 folds are used for the training data. Cross-validation estimates are generally pessimistically biased, as training is performed using a subsample of the available data.

\section{Results}

All subjects during preliminary testing were reported to be fully compliant in following given directions. Analysis of power spectra during full-screen checkerboard trials resulted in the selection of $17 \mathrm{~Hz}$ and $20 \mathrm{~Hz}$ as the bilateral checkerboard frequencies. These frequencies were employed in each of the four test trials for all subjects. Power spectra for fullscreen checkerboard trials for a representative subject are shown in Figure 1.

Note that peaks exist at both the frequency of modulation of each constituent square of the checkerboard (henceforth referred to as the first harmonic) and the alternation rate (second harmonic). Both the flicker stimulus and checkerboard SSVEP frequency effects described above are exhibited in the spectrum due to the large size of the constituent squares of the full-screen checkerboard pattern. As expected, the second harmonic was more dominant once the checkerboards were made smaller such that the pattern as a whole could be viewed in the subjects' foveal vision.

The power spectra for left and right gaze directions for a representative subject are shown in Figure 2. It can be seen that for this subject, the magnitude of the SSVEP response to a $17 \mathrm{~Hz}$ stimulus is greater than that for a $20 \mathrm{~Hz}$ stimulus, which demonstrates the need for classifier training to determine a decision threshold. Each subject's alpha rhythm caused little contamination of the spectra, being of low amplitude during testing - rapid stimulus presentation results in very little cortical idling in the visual cortex, and short trial length prevents arousal effects known also to affect alpha [15].

The classification accuracy for all five subjects using the two feature extraction methods are listed in Tables 1 and 2. Performance was assessed using both 1- and 2-second segments, and the question of whether inclusion (by averaging) of the first harmonic in the feature had any effect was 


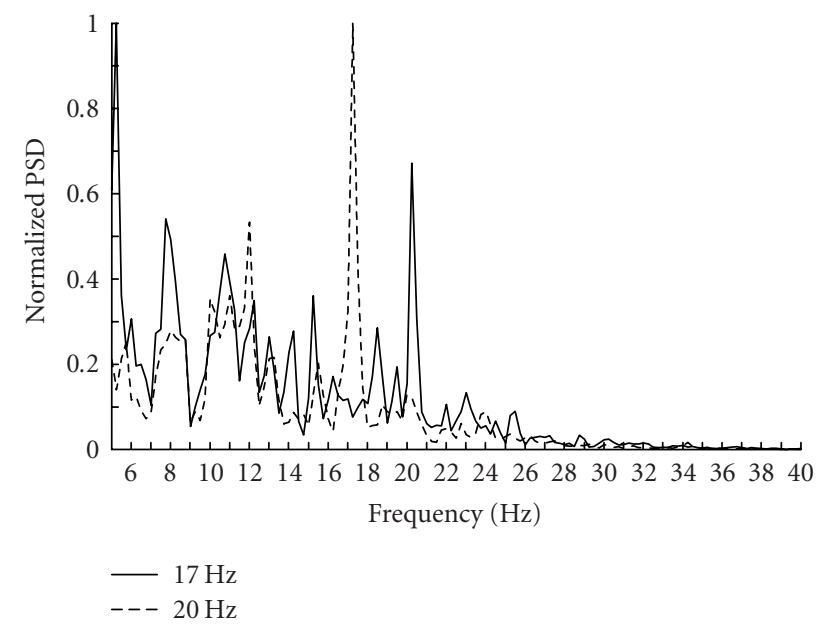

FIgURE 2: Power spectra for left and right gaze directions for subject 4. Spectra were calculated using the squared FFT method averaged across the entire 25-second trial.

TABLE 1: Offline performance for Method 1 averaged over two checkerboard configurations.

\begin{tabular}{|c|c|c|c|c|}
\hline \multirow{2}{*}{ Subject } & \multicolumn{2}{|c|}{ 2nd harmonic only } & \multicolumn{2}{|c|}{ 1st +2 nd harmonic } \\
\hline & 1s window & 2 s window & 1s window & 2s window \\
\hline Subject 1 & $88.4 \%$ & $92.2 \%$ & $79.3 \%$ & $88.2 \%$ \\
\hline Subject 2 & $72.2 \%$ & $79.0 \%$ & $70.4 \%$ & $74.3 \%$ \\
\hline Subject 3 & $58.7 \%$ & $62.0 \%$ & $62.4 \%$ & $69.3 \%$ \\
\hline Subject 4 & $75.7 \%$ & $81.4 \%$ & $67.4 \%$ & $72.9 \%$ \\
\hline Subject 5 & $57.0 \%$ & $54.2 \%$ & $52.1 \%$ & $50.8 \%$ \\
\hline Average across subjects & $70.4 \%$ & $74.4 \%$ & $66.3 \%$ & $71.1 \%$ \\
\hline
\end{tabular}

addressed. This results in the augmented feature

$$
F 1^{\prime}(n)=\log \left(\frac{\text { mean }\left(X_{n}(f 1), X_{n}(f 1 / 2)\right)}{\text { mean }\left(X_{n}(f 2), X_{n}(f 2 / 2)\right)}\right)
$$

for Method 1 and similarly for Method 2.

For both methods, analysis using 2-second segments is shown to perform better than 1-second segments. Also it can be seen that inclusion of the first harmonic in the augmented feature in fact degraded performance slightly. Performance of these two methods was comparable, with the more noiseresilient autocorrelation method performing marginally better as expected.

\section{REAL-TIME BCI GAME}

\subsection{Methods}

\section{(A) MindBalance-the game}

The object of the MindBalance game is to gain 1D control of the balance of an animated character on a tightrope using only the player's EEG. As mentioned in Section 1, the game involves the movement of the animated character within a virtual environment, with both the character and environment modelled as $3 \mathrm{D}$ volumes. The lighting and virtual camera position change in response to the character's movements within the environment. During the game, a musical soundtrack as well as spoken comments by the character are also played over the aforementioned speakers to make the game more engaging.

A checkerboard is positioned on either side of the character. These checkerboards are phase-reversed at 17 and $20 \mathrm{~Hz}$. A game begins with a brief classifier training period. This requires the subject to attend to the left and right checkerboards as indicated by arrows for a period of 15 seconds each. This process is repeated three times (Figure 3). During this training period, audio feedback is continually presented using speakers located behind the subject. The audio feedback is in the form of a looped double-click sound, the play speed of which is linearly related to the feature $(F 1$ in the case of Method 1 or F2 in the case of Method 2). Feedback is presented in order to ensure compliance during the critical training period.

In the game, the tightrope walking character walks towards the player and stumbles every $1.5-5.0$ seconds to one side chosen randomly. The player must intervene to shift the character's balance so that it remains stable on the tightrope. To do this, the player must direct his gaze and focus on the checkerboard on the opposite side of the screen to 
TABLE 2: Offline performance for Method 2 averaged over two checkerboard configurations.

\begin{tabular}{lcccc}
\hline \multirow{2}{*}{ Subject } & \multicolumn{2}{c}{ 2nd harmonic only } & \multicolumn{2}{c}{ 1st + 2nd harmonic } \\
\cline { 2 - 5 } & 1s window & 2s window & 1s window & $82.9 \%$ \\
Subject 1 & $89.8 \%$ & $96.1 \%$ & $73.2 \%$ & $89.1 \%$ \\
Subject 2 & $71.0 \%$ & $80.4 \%$ & $62.7 \%$ & $73 \%$ \\
Subject 3 & $61.7 \%$ & $65.8 \%$ & $71.9 \%$ & $78.9 \%$ \\
Subject 4 & $80.1 \%$ & $82.3 \%$ & $59.4 \%$ & $55.0 \%$ \\
Subject 5 & $59.5 \%$ & $62.0 \%$ & $70.0 \%$ & $75.5 \%$ \\
Average across subjects & $72.4 \%$ & $77.3 \%$ & & \\
\hline
\end{tabular}

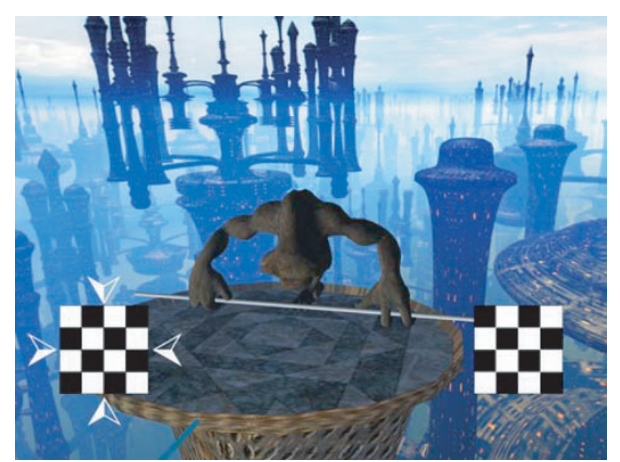

FIGURE 3: The training sequence.

which the character is losing balance (Figure 4). The character's off-balance animation lasts for 3 seconds. This duration was chosen to give the player time to realize which checkerboard required fixation to elicit the required SSVEPs and help the character regain his balance. At the end of the 3-second animation, a decision based on the most recent 1 or 2 seconds of EEG is obtained. To allow for better game play, a second more pronounced off-balance 3second animation was used in order to give a player a second chance in the case where an incorrect decision was obtained from the EEG. There was also an optional play mode where an EEG feature value within a certain range of the decision threshold, when detected at the end of the off-balance animation, resulted in no decision being taken and the original 3-second off-balance animation being simply replayed. This dead zone was removed during our online tests.

\section{(B) Signal processing and the C\# engine}

The overall processing system is shown in Figure 5. In order to carry out this study, a programming engine and platform were required, capable of rendering detailed 3D graphics while at the same time processing continuous EEG data to control a sprite within the game. This was accomplished using a combined graphics, signal processing, and network communications engine implemented in C\#. ${ }^{1}$ One machine

\footnotetext{
${ }^{1}$ Implemented by the MindGames Group at Media Lab Europe.
}

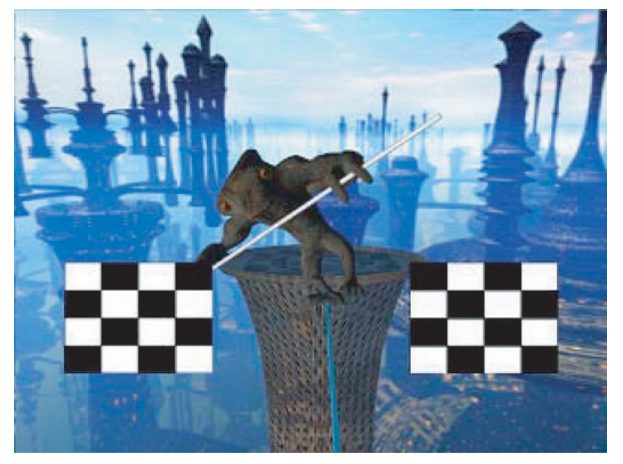

Figure 4: The character loses balance during the game.

is dedicated to the rendering of the $3 \mathrm{D}$ graphics while a second machine was dedicated to the real-time data acquisition and signal processing of the EEG data. This signal processing engine allows selection of signal processing functions and parameters as objects to be included into a chain of signal processing blocks to perform the required processing. Whenever a decision on the fate of the animated character is required, a request in the form of a UDP packet is sent over the local area network to the signal processing machine which sends back a decision based on the most recent feature extracted from the EEG.

\section{(C) Interface equipment of game control}

The setup for the real-time BCI game was similar to that used in the preliminary offline analysis. One difference was the amplification stage in which the Grass Telefactor P511 rack amplifiers were replaced by Biopac biopotential amplifiers.

The subject was seated in front of a large screen on which a $140 \times 110 \mathrm{~cm}$ image was projected. Within the game pictured in Figures 3 and 4, each $4 \times 4$ checkerboard pattern's medial edge was situated $8.5^{\circ}$ bilateral to the tightrope, centered on the horizontal meridian, and subtended a visual angle of $11.4^{\circ}$ vertically and $11.8^{\circ}$ horizontally.

\section{(D) Subjects and test protocol}

Six male subjects aged between 24 and 34 participated in the following test procedure to assess performance of the realtime BCI game. All subjects had normal or corrected-tonormal vision. 


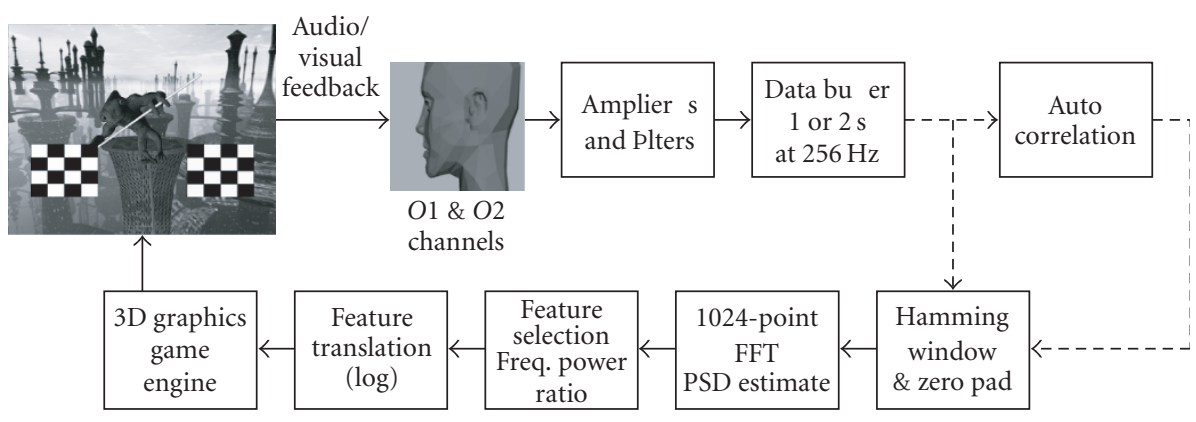

FIGURE 5: Flowchart of signal processing stages employed in real-time BCI game.

TABle 3: Percentage of correct decisions in real-time game play, using Method 1 with second SSVEP harmonic only.

\begin{tabular}{lcc}
\hline Subject & 1s window & 2s window \\
\hline Subject 1 & $75.0 \%$ & $100 \%$ \\
Subject 2 & $72.7 \%$ & $100 \%$ \\
Subject 3 & $75.0 \%$ & $70.6 \%$ \\
Subject 4 & $69.2 \%$ & $100 \%$ \\
Subject 5 & $87.5 \%$ & $78.2 \%$ \\
Subject 6 & $100 \%$ & $88.2 \%$ \\
Average across subjects & $79.9 \%$ & $89.5 \%$ \\
\hline
\end{tabular}

Each subject was asked to play the game eight times. Four of the games were played where the EEG was analyzed by the FFT method described above as Method 1 for the offline data. In two of these games, the decision on the fate of the tightrope walking character was based on a 1-second window of EEG data, and in the other two games, the decision was based on a 2 -second window. The other four games were played using EEG analyzed by Method 2, the autocorrelation followed by FFT method. Again, two games used 1second segments of EEG data and two games used 2-second segments.

On average, there were eight trials per game. This varied from game to game as a result of the random number of steps taken by the character between losses of balance and the fact that in seven of the 48 games played, two consecutive errors occurred resulting in the character falling from the tightrope and the end of the game.

\section{Results}

Tables 3 and 4 list the percentage of correct decisions resulting in the desired regain of balance on the tightrope. In seven of the 48 games played, two consecutive errors occurred resulting in the character falling from the tightrope, causing the game to end. Three of the six subjects did not allow the character to fall off the tightrope in any of the eight games.

One objective measure of BCI performance is the bit rate, as defined by Wolpaw [16]. For a trial with $N$ possible symbols in which each symbol is equally probable, the probability $(P)$ that the symbol will be selected is the same for each
TABle 4: Percentage of correct decisions in real-time game play, using Method 2 with second SSVEP harmonic only.

\begin{tabular}{lcc}
\hline Subject & 1s window & 2s window \\
\hline Subject 1 & $87.5 \%$ & $91.7 \%$ \\
Subject 2 & $50.0 \%$ & $58.3 \%$ \\
Subject 3 & $85.7 \%$ & $46.2 \%$ \\
Subject 4 & $85.7 \%$ & $75.0 \%$ \\
Subject 5 & $63.6 \%$ & $100 \%$ \\
Subject 6 & $87.5 \%$ & $92.3 \%$ \\
Average across subjects & $76.7 \%$ & $77.3 \%$ \\
\hline
\end{tabular}

symbol, and each error has the same probability, then the bit rate can be calculated as follows:

Bits per symbol $=\log _{2} N+P \cdot \log _{2} P+(1-P) \cdot \log _{2} \frac{1-P}{N-1}$,

Bit Rate $=$ bits per symbol $*$ symbols per minute.

In the case of the present study, one symbol is sent per trial. Using this definition of bit rate and given that each trial lasts for 3 seconds and the peak accuracy for the real-time system is $89.5 \%$, the bit rate is $10.3 \mathrm{bits} / \mathrm{min}$.

\section{DISCUSSION}

The results from this study indicate that the distinct SSVEP responses elicited by phase-reversing checkerboard patterns can be successfully used to make binary decisions in real time in a BCI-controlled game involving a visually elaborate environment.

The two feature extraction methods can be directly compared for the offline data, given that the methods were used to classify the same data set. The results for both methods are comparable, with Method 2 performing marginally better than Method 1. This may be due to the resilience of Method 2 to uncorrelated noise.

In the real-time gaming situation, Method 1 and Method 2 were employed during separate games. Therefore, classification for the two methods was performed on different data sets. For this reason, and because each subject undertook 
a relatively small number of trials, a direct comparison between the methods in the real-time tests is not as meaningful. The fact that Method 1 performs better than Method 2 may be attributable more to the anomalous performance of subjects 2 and 3 during the games played using Method 2 than to the feature extraction method itself.

In both online and offline testings, classification based on 2-second windows exceeded that of 1-second windows for all features. This is to be expected as a 2 -second window gives higher-frequency resolution and allows more accurate extraction of the SSVEP peak amplitudes. As mentioned earlier, a bit rate $10.3 \mathrm{bits} / \mathrm{min}$ is achievable using the full trial length of 3 seconds, allowing for the time taken for the subject to respond to the loss of balance of the character in the game and for the elicitation of the SSVEP. It is also useful to calculate theoretical bit rate maxima based purely on the 1- and 2-second EEG windows. This gives a peak bit rate of $15.5 \mathrm{bits} / \mathrm{min}$ for the 2 -second window and $16.6 \mathrm{bits} / \mathrm{min}$ for the 1-second window. It is worth noting that the bit rate defined in (6) is designed to encourage accuracy over speed and as a result, the penalty incurred by the drop in accuracy almost negates the doubling of the number of symbols per minute achieved using the 1-second window.

The decrease in performance obtained by the inclusion of the first harmonic in the offline testing may be attributed to noise added to the first harmonic due to activity in the alpha band. It was for this reason that the frequencies of the stimuli were originally chosen outside the alpha range and only the second harmonic was used in the real-time testing.

Two additional interesting observations were made during both the offline and online testings. Firstly, the two investigators who themselves participated as subjects in the study achieved better performance both in terms of accuracy in the offline analysis and in terms of success in completing the game. This implies that either practice or a more motivated approach to stimulus fixation results in a more pronounced visual response. This may be thought of in terms of visual attention. Endogenous modulation of SSVEP response has been reported as possible in relation to both foveal fixated stimuli [10] and covertly attended stimuli in peripheral vision [17]. The improved discriminability of the SSVEP with increased "conscious effort" may be related to the ability of the subject to focus selective attention on the fixated stimulus, as well as the ability to inhibit processing of distractors in the peripheral visual field.

Secondly, in post-experiment debriefing, subjects reported that audio feedback during training aided in the successful sustained fixation on a particular stimulus and the inhibition of responses to distractions. Also, in the case of an error causing the character to drop to the second level of imbalance, subjects found it possible to adjust their fixation strategy, most notably through observing the checkerboard as a whole rather than specifically fixating on any individual elements or allowing perception of the phase reversal as a moving pattern. These adjustments in fixation strategy prompted by the discrete presentation of biofeedback during the game in conjunction with the motivation to succeed in the task evoked by the immersive environment may be the reason for the better average performance during the realtime sessions (peak 89.5\%) when compared with the offline results (peak $75.5 \%$ ).

A possible explanation for the high performance of this BCI design in spite of continuous distracting stimulation may be offered by considering the underlying physiology. The topographic organization of the primary visual cortex is such that a disproportionately wide cortical area is devoted to the processing of information from the central or foveal region of the visual field, and thus directing one's gaze at a desired repetitive stimulus produces an SSVEP response to which all other responses to competing stimuli are small in comparison.

The SSVEP BCI design has not been actively employed in alternative or augmentative communication (AAC) for the disabled. This is partly due to the fact that, for successful operation, the subject's ocular motor control must be fully intact to make selections by shifting gaze direction. Given the range of accessibility options available for the disabled, it is only in very extreme cases, such as those where reliable eye movement is not possible, that a communication medium driven by EEG generated by the brain itself is applicable.

While the need for reliable ocular motor control is a prerequisite for using the $\mathrm{BCI}$ described in this paper, we speculate that the use of the BCI to control a character in an engaging game such as that described may prove a useful tool in assisting with motivational issues pertaining to ALS patients. As BCI systems take considerable training to master, typically several months, this system may serve to encourage patients to train for a greater length of time. It may also be possible that through continued and regular playing of the game, an ALS patient may be able to retain an acceptable level of control, even after ocular motor control has deteriorated to the point where eye-tracking systems are no longer feasible. This would involve detection of changes in the amplitudes of the SSVEP as modulated by attention to the stimuli in one's peripheral vision. In order to explore this idea, the authors are currently extending this study to covert visual attention, in which subjects direct attention to one of two bilateral stimuli without eye movement.

Also worthy of investigation is the presentation of more stimuli in order to give multidimensional control in the $3 \mathrm{D}$ environment.

\section{CONCLUSION}

This paper presented the application of an effective EEGbased brain-computer interface design for binary control in a visually elaborate immersive 3D game. Results of the study indicate that successful binary control using steady-state visual evoked potentials is possible in an uncontrolled environment and is resilient to any ill effects potentially incurred by a rich detailed visual environment. All six subjects demonstrated reliable control achieving an average of $89.5 \%$ correct selections for one of the methods investigated, corresponding to a bit rate of $10.3 \mathrm{bits} / \mathrm{min}$. 


\section{ACKNOWLEDGMENTS}

We wish to acknowledge Phil McDarby for his assistance in designing the gaming environment. We would also like to thank the subjects who participated in the experimental sessions.

\section{REFERENCES}

[1] J. R. Wolpaw, N. Birbaumer, D. J. McFarland, G. Pfurtscheller, and T. M. Vaughan, "Brain-computer interfaces for communication and control," Clinical Neurophysiology, vol. 113, no. 6, pp. 767-791, 2002.

[2] L. A. Farwell and E. Donchin, "Talking off the top of your head: toward a mental prosthesis utilizing event-related brain potentials," Electroencephalography and Clinical Neurophysiology, vol. 70, no. 6, pp. 510-523, 1988.

[3] E. E. Sutter, "The visual evoked response as a communication channel," in Proc. IEEE/NSF Symposium on Biosensors, pp. 95100, Los Angeles, Calif, USA, September 1984.

[4] N. Birbaumer, A. Kubler, N. Ghanayim, et al., "The thought translation device (TTD) for completely paralyzed patients," IEEE Trans. Rehab. Eng., vol. 8, no. 2, pp. 190-193, 2000.

[5] G. Pfurtscheller and F. H. Lopes da Silva, "Event-related EEG/MEG synchronization and desynchronization: basic principles," Clinical Neurophysiology, vol. 110, no. 11, pp. 1842-1857, 1999.

[6] G. Pfurtscheller and C. Neuper, "Motor imagery and direct brain-computer communication," Proc. IEEE, vol. 89, no. 7, pp. 1123-1134, 2001.

[7] J. R. Wolpaw, D. J. McFarland, G. W. Neat, and C. A. Forneris, "An EEG-based brain-computer interface for cursor control," Electroencephalography and Clinical Neurophysiology, vol. 78, no. 3, pp. 252-259, 1991.

[8] D. Regan, Human Brain Electrophysiology: Evoked Potentials and Evoked Magnetic Fields in Science and Medicine, Elsevier, New York, NY, USA, 1989.

[9] M. Cheng, X. Gao, S. Gao, and D. Xu, "Design and implementation of a brain-computer interface with high transfer rates," IEEE Trans. Biomed. Eng., vol. 49, no. 10, pp. 1181-1186, 2002.

[10] M. Middendorf, G. R. McMillan, G. L. Calhoun, and K. S. Jones, "Brain-computer interfaces based on the steady-state visual-evoked response," IEEE Trans. Rehab. Eng., vol. 8, no. 2, pp. 211-214, 2000.

[11] K. E. Misulis, Spehlmann's Evoked Potential Primer, Butterworth-Heinemann, Boston, Mass, USA, 1994.

[12] F. Sharbrough, G.-E. Chatrian, R. P. Lesser, H. Lüders, M. Nuwer, and T. W. Picton, "American electroencephalographic society guidelines for standard electrode position nomenclature," Clinical Neurophysiology, vol. 8, no. 2, pp. 200-202, 1991.

[13] G. R. Burkitt, R. B. Silberstein, P. J. Cadusch, and A. W. Wood, "Steady-state visual evoked potentials and travelling waves," Clinical Neurophysiology, vol. 111, no. 2, pp. 246-258, 2000.

[14] B. D. Ripley, Pattern Recognition and Neural Networks, Cambridge University Press, Cambridge, UK, 1996.

[15] W. Klimesch, "EEG alpha and theta oscillations reflect cognitive and memory performance: a review and analysis," Brain Research Reviews, vol. 29, no. 2-3, pp. 169-195, 1999.

[16] J. R. Wolpaw, H. Ramoser, D. J. McFarland, and G. Pfurtscheller, "EEG-based communication: improved accuracy by response verification," IEEE Trans. Rehab. Eng., vol. 6, no. 3, pp. 326-333, 1998.
[17] S. T. Morgan, J. C. Hansen, and S. A. Hillyard, "Selective attention to stimulus location modulates the steady-state visual evoked potential," Proceedings of the National Academy of Sciences of the United States of America, vol. 93, no. 10, pp. 4770$4774,1996$.

E. C. Lalor received the B.E. degree in electronic engineering from University College Dublin, Ireland, in 1998 and the M.S. degree in electrical engineering from the University of Southern California in 1999. He is currently working towards the Ph.D. degree in the Department of Electronic and Electrical Engineering in University College Dublin, Ireland. From 2002 to 2005, he worked as a Research Asso-

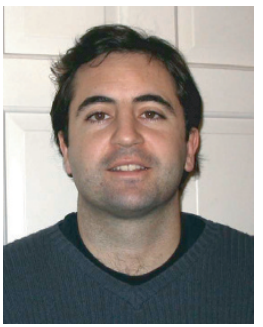
ciate with Media Lab Europe, the European research partner of the MIT Media Lab. His current interests include braincomputer interfaces and signal processing applications in neuroscience.

S. P. Kelly received the B.E. degree in electronic engineering and $\mathrm{Ph} . \mathrm{D}$ degree in biochemical engineering from University College Dublin, Ireland, in 2001 and 2005, respectively. He is currently a Postdoctoral Research Fellow in the Cognitive Neurophysiology Laboratory, Nathan S. Kline Institute for Psychiatric Research in New York. His current research interests include the neurophysiology of selective attention and multisensory integration in humans, and EEG-based braincomputer interfacing for alternative communication and control.

C. Finucane was born in Dublin in 1979. $\mathrm{He}$ graduated from University College Dublin (UCD) in 2001 with a B.S. degree in electronic engineering. He subsequently completed an M. Eng. Sc. degree at UCD and the National Rehabilitation Hospital for work entitled "EEG-based brain-computer interfaces for the disabled" in 2003 before joining the Department of Medical Physics, St. James's Hospital, Dublin, where he cur-

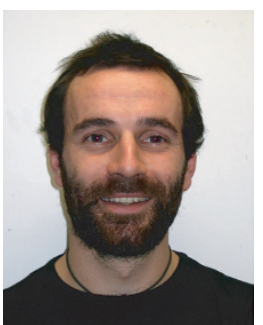
rently works as a Medical Physicist. Finucane's research interests include the development of novel brain-computer interfaces, neurophysiological signal analysis, biomedical applications of multimedia, wireless and Internet technologies, and biological systems modelling.

R. Burke received the B.S. Eng. degree in mathematics and engineering from Queen's University, Kingston, Canada, in 1999, and an SM degree in media arts and science from the Massachusetts Institute of Technology in 2001. From 2002 to 2004, he worked as a Research Associate with the MindGames Group at Media Lab Europe, Dublin. He is currently a Member of the Developer and Platform Group at Microsoft.

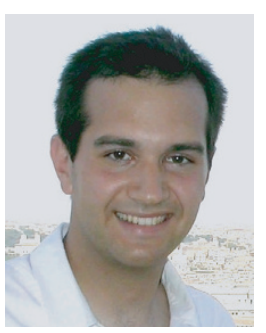


R. Smith obtained the B.E. degree in electronic engineering from University College Dublin (UCD), Ireland, in 2002. He subsequently completed an M. Eng. Sc. at UCD for research that focused on neurophysiological signal processing and the development of brain-computer interfaces. His research primarily focuses on EEG-based BCIs and their possibilities in the field of neurological rehabilitation.

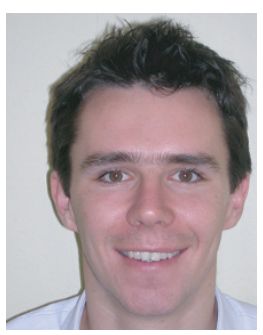

R. B. Reilly received his B.E., M. Eng. Sc., and Ph.D. degrees in 1987, 1989, and 1992, all in electronic engineering, from the $\mathrm{Na}$ tional University of Ireland. Since 1996, he has been on the academic staff in the Department of Electronic and Electrical Engineering at University College Dublin. $\mathrm{He}$ is currently a Senior Lecturer and researches into neurological signal processing and multimodal signal processing. He was

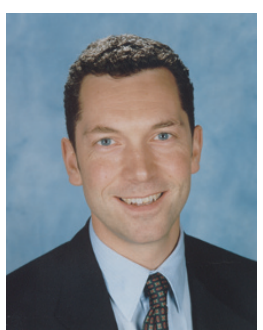
the 1999/2001 Silvanus P. Thompson International Lecturer for the IEE. In 2004, he was awarded a US Fulbright Award for research collaboration into multisensory integration with the Nathan S. Kline Institute for Psychiatric Research, New York. He is a reviewer for the Journal of Applied Signal Processing and was Guest Editor for the mini issue on multimedia human-computer interface, September 2004. He is the Republic of Ireland Representative on the Executive Committee of the IEEE United Kingdom and Republic of Ireland Section. He is an Associate Editor for IEEE Transactions on Multimedia and also a reviewer for IEEE Transactions on Biomedical Engineering, IEEE Transactions on Neural Systems and Rehabilitation Engineering, IEEE Transactions on Industrial Electronics, Signal Processing, and IEE Proceedings Vision, Image \& Signal Processing.

G. McDarby obtained the B.E. and M.S. degrees in electronic engineering from University College Dublin, Ireland, in 1988 and 1995, respectively. He received the Ph.D. degree in biomedical signal processing in 2000 from the University of New South Wales, Sydney. Since 2000, he has worked as a principal Research Scientist in Media Lab Europe leading a multidisciplinary group called MindGames. His research is focused

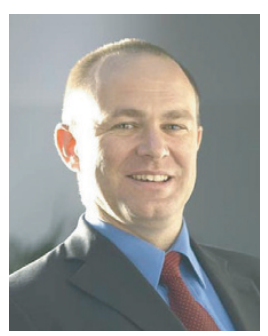
on combining sensory immersion (augmented reality), game play, novel biometric interfaces, and intelligent biofeedback to constructively affect the state of the human mind. He is strongly committed to finding ways where technology can be a transformational tool to people marginalized in society and is heavily involved with the Intel Computer Clubhouse Programme. He is a much sought-after speaker on technology and philosophy and has recently been nominated to the European Academy of Sciences for contributions to human progress. 